\title{
Polifenoles totales en pan de abeja (Apis mellifera L.) de colmenas de la Región de La Araucanía
}

\author{
Total polyphenols in bee bread (Apis mellifera L.) \\ from hives the Araucanía Region \\ Ximena Araneda Durán ${ }^{1 *}$, Ingrid Quezada Mardones ${ }^{2}$, \\ María Martínez Gutiérrez y Daniza Morales Ulloa ${ }^{1}$
}

\section{RESUMEN}

\begin{abstract}
El pan de abeja es polen almacenado parcialmente fermentado que las obreras de Apis mellifera L. recolectan y lo mezclan con sus propias enzimas digestivas, haciéndolo un alimento sano y nutritivo para ellas debido a las propiedades funcionales. Por esta razón, el objetivo de este estudio fue determinar la concentración de polifenoles presentes en pan de abeja provenientes de dos sectores de la Región de La Araucanía mediante dos métodos de extracción. Para ello se obtuvieron cinco panales con pan de abeja del sector de Pillanlelbún en la comuna de Lautaro y el sector de Pidenco-Codihue comuna de Carahue. El total de compuestos fenólicos se analizó colorimétricamente por medio del método de Folin Ciocalteu en el Laboratorio de Bromatología de la Universidad Católica de Temuco. La mayor concentración de polifenoles se observó en la localidad de Pillanlelbún, tanto para la extracción con metanol

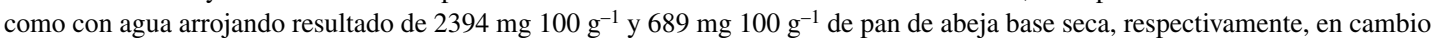

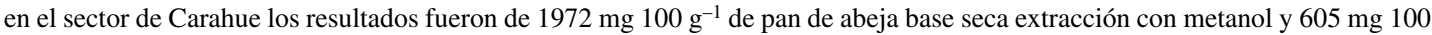
$\mathrm{g}^{-1}$ de pan de abeja base seca extracción con agua. Esta diferencia estaría dada por la composición floral de los pólenes de cada sector, siendo más abundante en polifenoles en el sector de Pillanlelbún.
\end{abstract}

Palabras clave: polen, método de extracción, Carahue, Pillanlelbún.

\begin{abstract}
The bee bread stored pollen fermentation is that workers of Apis mellifera L. collected and mixed with their own digestive enzymes, making it a healthy and nutritious food for them due to the functional properties. For this reason, the aim of this study was to determine the concentration of polyphenols present in bee bread from two sectors of the Araucanía Region using two extraction methods. To do this, five combs with bee bread were obtained from the Pillanlelbún sector of the district of Lautaro and from the Pidenco-Codihue sector of the district of Carahue. The total phenolic compounds were subjected to colorimetric analysis using the Folin Ciocalteu method in the Bromatology Laboratory of the Catholic University of Temuco. A greater concentration of polyphenols was observed in the Pillanlelbún sector, for extraction both with methanol and with water, giving a result of $2394 \mathrm{mg}^{100 \mathrm{~g}^{-1}}$

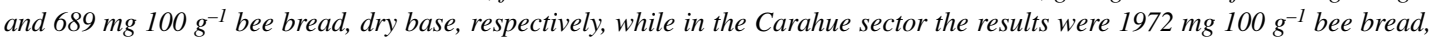
dry base, for extraction with methanol and $605 \mathrm{mg} 100 \mathrm{~g}^{-1}$ bee bread, dry base, for extraction with water. This difference would arise from the composition of the flora from which the pollen, with polyphenols being more abundant in the Pillanlelbuin sector.

Key words: pollen, extraction method, Carahue, Pillanlelbún.
\end{abstract}

\section{Introducción}

El pan de abeja es polen almacenado parcialmente fermentado (Bogdanov, 2004) que las obreras recolectan y lo mezclan con sus propias enzimas digestivas (Nagai et al., 2004) y que almacenan en las celdillas agregando una capa de miel como única fuente de carbono (Audisio et al., 2005). Esto, al quedar sellado y en los panales adyacentes a la cámara de cría $\left(35^{\circ} \mathrm{C}\right.$ a $\left.36^{\circ} \mathrm{C}\right)$, sufre una serie de transformaciones, debido a la acción de diferentes enzimas, microorganismos, humedad y temperatura que permiten la conversión, mejoramiento y preservación del polen almacenado (Gilliam, 1997), la cual, después de dos semanas se convierte en pan de abeja (Mutsaers y Kat-Reynen, 2005).

\footnotetext{
1 Universidad Católica de Temuco, Facultad de Recursos Naturales, Escuela de Agronomía. Rudecindo Ortega 02950, Temuco. Chile.

2 Servicio Agrícola y Ganadero (SAG). Servicio Agrícola Ganadero (SAG), San José 242, Puerto Varas.

* Autor por correspondencia: xaraneda@uct.cl
}

Fecha de Recepción: 21 Agosto, 2013.

Fecha de Aceptación: 15 Noviembre, 2013. 
Este polen, que es la principal fuente de proteína para las abejas (Almeida-Muradian et al., 2005), proviene de frutas, hortalizas y cereales, los cuales pueden biosintetizar bioactivos como polifenoles, compuestos con más de 500 moléculas con diferentes propiedades y biodisponibilidades (Manach et al., 2004; Rubilar et al., 2006; Neveu et al., 2010; Tresserra-Rimbau et al., 2013), que pueden ser transferidos a este (Baltrušaityté et al., 2007), y que utilizan para la elaboración del pan, haciéndolo un alimento sano y nutritivo para ellas, debido a las propiedades funcionales y contenido de flavonoides presentes en este, correlacionados con su origen floral (Nagai et al., 2004; Yao et al., 2004; Stanciu et al., 2008) existiendo, además, en algunos de sus compuestos fenólicos propiedades antioxidantes (Stanciu et al., 2007).

En este contexto es importante determinar la concentración de polifenoles totales de este producto elaborado por las abejas, en diferentes localidades de la Región de La Araucanía mediante dos métodos de extracción con el propósito de verificar diferencias, según origen de colecta de polen y técnicas de análisis.

\section{Materiales y Métodos}

Las muestras de pan de abeja fueron colectadas de dos localidades de la Región de La Araucanía, sector de Pillanlelbún, a $18 \mathrm{~km}$ al norte de Temuco, comuna de Lautaro (38 $39^{\circ} 17,2^{\prime}$ " lat. sur, $72^{\circ} 26^{\prime} 56^{\prime \prime}$ long. oeste) y el sector de Pidenco-Codihue comuna de Carahue (39 $43^{\prime}$ lat. sur; $74^{\circ} 11^{\prime}$ long. oeste). Para ello se obtuvieron cinco panales con pan de abeja de la temporada primavera-verano, los cuales fueron trasladados en bolsas plásticas y congelados para su análisis posterior en el Laboratorio de Bromatología de la Escuela de Agronomía de la Universidad Católica de Temuco.

Para la extracción del pan de abeja de los panales se tomó desde la cámara de cría un panal con polen de cada colmena (seis colmenas por sector) los que fueron llevados hasta el laboratorio en una caja térmica y refrigerados hasta su utilización posterior. Para retirar el polen de las celdillas estas se rompieron con una espátula. El material obtenido se colocó en un mortero de loza y se homogenizó.

Para el ensayo se utilizaron dos tratamientos, el primer tratamiento consistió en diluir $0,5 \mathrm{~g}$ de pan de abeja en agua destilada y para el segundo tratamiento se diluyeron $0,5 \mathrm{~g}$ en metanol al $80 \%$.
Se realizaron seis repeticiones por tratamiento. Las repeticiones consistieron en pan de abejas colectado de seis colmenas por cada sector.

El total de compuestos fenólicos se analizó colorimétricamente por medio del método de Folin Ciocalteu modificado por Singleton y Rossi (1965). Con un valor de 2,5 ml de Folin Ciocalteu, el reactivo se diluyó 10 veces, se utilizaron $2 \mathrm{ml}$ de carbonato de sodio al 75\%; 0,5 g de pan de abeja diluido en agua y metanol al 80\%. La absorbancia se midió a $765 \mathrm{~nm}$ después de 15 minutos de haber recibido una temperatura de $50^{\circ} \mathrm{C}$. Como blanco se utilizó una mezcla de agua y reactivos. La concentración de polifenoles se expresó en equivalentes de ácido gálico (EAG).

El análisis polínico del pan de abeja consistió en realizar un catastro de la flora presente en los lugares donde se extrajeron las muestras. Para ello se realizó un muestreo aleatorio simple recorriendo $3 \mathrm{~km}$ a la redonda de donde estaban ubicados los apiarios. El muestreo se realizó durante octubre recolectando e identificando las especies presentes en el lugar (clasificándolas por familia) y luego las muestras fueron analizadas en el laboratorio. El análisis consistió en extraer polen de las especies recolectadas, fijarlas sobre un portaobjeto, aplicarle una solución de gelatina con glicerol y safranina, para teñir el polen, luego se identificó cada una de las especies recolectadas y se observaron en el microscopio, posteriormente se diluyó $0,2 \mathrm{~g}$ de pan de abeja, en $20 \mathrm{ml}$ de agua. Se agitaron en un agitador magnético por 15 minutos, seguidamente se colocó una gota de esta solución en un portaobjeto y se utilizó la misma solución para teñir. Posteriormente, se analizó en el microscopio comparándola con los pólenes de las especies recolectadas y apoyada en bibliografía.

Se utilizó un factorial de 2 × 2 (dos zonas de colecta y dos métodos de extracción). Los resultados obtenidos de cada parámetro evaluado (contenido de polifenoles y composición de polen presente en pan de abeja) en la investigación fueron examinados mediante un análisis de varianza (ANDEVA) $(\mathrm{p} \leq 0,05)$, los resultados expresados como porcentajes fueron transformados utilizando el arcoseno de la raíz del porcentaje, y cuando correspondió se realizó la comparación de medias mediante el test de comparación múltiple de Tuckey $(\mathrm{p} \leq 0,05)$. Para el análisis estadístico se utilizó el programa estadístico SPSS (Statistical Product and Service Solutions) versión 15.0 para Windows (SPSS Inc, 2006). 


\section{Resultados y Discusión}

Los resultados indican que en todas las muestras existe presencia de polifenoles, los cuales varían en su concentración tanto por el tipo de extracción como por su ubicación geográfica (Tabla 1). Tal como lo señalan Mărghitaş et al. (2009), quienes indican que existe una gran variabilidad en relación con la correspondencia entre la actividad antioxidante y el contenido de polifenoles totales del polen con diferentes orígenes botánicos.

Según los resultados, estos señalan que existen diferencias estadísticas $(\mathrm{p} \leq 0,05)$ para los sectores de Pillanlelbún y Carahue, obteniéndose una mayor concentración de polifenoles en ambos sectores estudiados mediante la extracción con metanol que con agua. Por otra parte, al comparar las concentraciones de polifenoles según el sector de origen, los resultados también señalan que existen diferencias significativas, produciéndose una mayor concentración en el sector de Pillanlelbún (1541 mg $100 \mathrm{~g}^{-1}$ de pan de abeja base seca) que en el de Carahue (1289 mg $100 \mathrm{~g}^{-1}$ de pan de abeja base seca) (Tabla 1).

Estos resultados coinciden con Stanciu et al. (2007), quienes al comparar diferentes métodos de extracción de polifenoles en pan de abeja comprobaron que la extracción con metanol resultó ser la que obtuvo la mayor cantidad de fenoles con 22,72 mg EAG g ${ }^{-1}$, lo que concuerda con estudios realizados por Rubilar et al. (2006), quienes obtuvieron como resultado que el mayor rendimiento de compuesto extraíble a partir de hojas de murta (Ugni molinae) corresponde a metanol (44\%), seguido muy cerca por el etanol $(40 \%)$ y agua (34\%).

Además, estos resultados también coinciden con lo expuesto por Carpes et al. (2007), quienes señalan que el importe total de compuestos fenólicos no solo varía con diferentes condiciones de extracción, sino que también varía en localidades diferentes. Tal como lo obtenido por Baltrušaityté et al. (2007), quienes indican que las concentraciones de polifenoles presentes varían según el origen del polen, es decir, con los diferentes tipos de flores de las cuales fueron recolectadas, lo cual depende claramente de la flora y la ubicación geográfica de esta. En este mismo sentido Stanciu et al. (2008) indican una gran variabilidad en relación con la correspondencia entre la actividad antioxidante y el contenido de polifenoles totales y flavonoides en el origen botánico de diferentes pólenes.

Con relación a la diferencia de la cantidad de polifenoles totales encontrados en pan de abeja entre el sector de Pillanlelbún y Carahue, este está dado principalmente por la composición del polen presente en pan de abeja (Figura 1A y 1B), ya que existe una gran variabilidad en el contenido

Tabla 1. Comparación de la concentración de polifenoles presente en pan de abeja (mg $100 \mathrm{~g}^{-1}$ de pan de abeja base seca), entre los sectores de Pillanlelbún y Carahue, mediante dos métodos de extracción.

\begin{tabular}{llll}
\hline \multirow{2}{*}{ Sector } & \multicolumn{2}{c}{ Método de extracción } & \multirow{2}{*}{ Promedio sector } \\
\cline { 2 - 3 } & Metanol & Agua & \\
\hline Pillanlelbún & $2394 \mathrm{Aa}$ & $689 \mathrm{Ab}$ & $1541 \mathrm{~A}$ \\
Carahue & $1972 \mathrm{Ba}$ & $605 \mathrm{Bb}$ & $1289 \mathrm{~B}$ \\
Promedio método de extracción & $2183 \mathrm{a}$ & $647 \mathrm{~b}$ & \\
\hline
\end{tabular}

Valores seguidos por distintas letras mayúsculas en las columnas o seguidos por distintas letras minúsculas en las filas presentan diferencias significativas según Test de Tukey $(\mathrm{p} \leq 0,05)$.
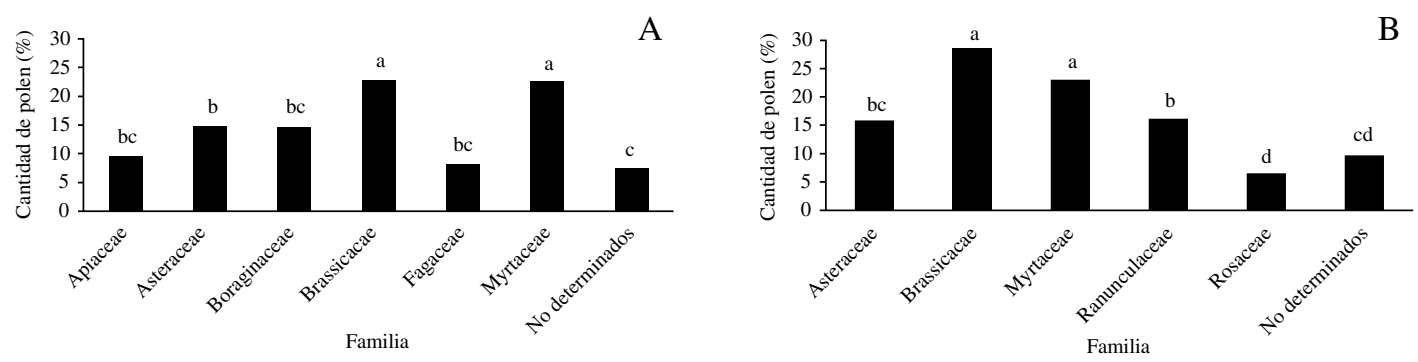

Figura 1. Porcentaje de participación de familias en la composición del polen presente en pan de abeja. A) Sector de Carahue. B) Sector de Pillanlelbún. Letras diferentes en barras indican diferencias significativas según test de Tuckey $(\mathrm{p}<0,05)$. 
de compuestos fenólicos en el polen de diferentes especies (Almeida-Muradian et al., 2005; Leja et al., 2007). En este sentido, la localidad de Pillanlelbún obtuvo los mayores contenidos de polifenoles en las muestras de pan de abeja que el sector de Carahue, lo que probablemente estaría dado por la presencia de especies de la familia Rosaceae como zarzamora (Rubus ulmifolius Schott.), al ser una rica fuente de compuestos polifenólicos (Manach et al., 2004), ya que la participación total de fenoles difieren considerablemente (Stanciu et al., 2008).

A pesar de existir diferencias estadísticamente significativas $(\mathrm{p} \leq 0,05)$ en el porcentaje de participación de especies en la composición del polen en pan de abeja (Figuras 1A y 1B), en ambos sectores la vegetación presente estuvo dada principalmente por especies de la familia Brasicaceae y Myrtaceae (Eucaliptus sp.), que también aportarían contenidos de polifenoles, ya que según estudios realizados por Isaza (2007), los polifenoles están distribuidos en varios sectores del reino de las plantas superiores, especialmente en las familias Leguminosae, Rosaceae, Polygonaceae, Fagaceae, Rhyzophoraceae, Myrtaceae y Melastomatacae.

\section{Conclusiones}

De acuerdo con los resultados obtenidos, se puede concluir que la concentración de polifenoles en pan de abeja varía según la localidad del sector de donde son tomadas las muestras al igual que el método de extracción, siendo mayor en el sector de Pillanlelbún con la extracción con metanol, debido probablemente al porcentaje de participación de diferentes familias en la composición del polen presente en pan de abeja.

\section{Literatura Citada}

Almeida-Muradian, L.B.; Pamplona, L.C.; Coimbra, S.; Barth, O.M.

2005. Chemical composition and botanical evaluation of dried bee pollen pellets. Journal of Food Composition and Analysis 18(1): 105-111.

Audisio, M.C.; Terzolo, H.R.; Apella, M.C.

2005. Bacteriocin from honeybee beebread Enterococcus avium, active against Listeria monocytogenes. Applied and Environmental Microbiology 71(6): 3373-3375.

Baltrušaityté, V.; Rimantas, P.; Čeksteryte, V.

2007. Radiacal scavenging activity of different floral origin honey and beebread phenolic extracts. Food Chemistry 101: 502-514.

Bogdanov, S.

2004. Quality and standars of pollen and beeswax. Apiacta 38: 334-341.

Carpes, S.; Begnini, R.; de Alencar, S.; Masson, M.

2007. Study of preparations of bee pollen extracts, antioxidant and antibacterial activity. Ciência e Agrotecnologia 31(6): 1818-1825.

Gilliam, M.

1997. Identification and roles of non-pathogenic microflora associated. FEMS Microbiology Letters 155(1): 1-10.

Isaza, J.

2007. Taninos o polifenoles vegetales. Scientia Et Tecnica 13(033): 13-18.

Leja, M.; Mareczek, A.; Wyzgolik, G.; Klepacz-Baniak, J.; Czekonska, K.

2007. Antioxidative properties of bee pollen in selected plant species. Food Chemistry 100: 237-240.

Manach, C.; Scalbert, A.; Morand, C.; Rémésy, C.; Jiménez, L. 2004. Polyphenols: food sources and bioavailability. American Journal of Clinical Nutrition's 79: 727-747.
Mărghitaș, L.; Stanciu, O.; Dezmirean, D.; Bobiș, O.; Popescu, O.; Bogdanov, E.; Campos, M.

2009. In vitro antioxidant capacity of honeybee-collected pollen of selected floral origin harvested from Romania. Food Chemistry 115(3): 878-883.

Mutsaers, M.; Kat-Reynen, C.

2005. Bee products: properties, processing and marketing. Agrodok, 42. Agromisa Foundation. Wageningen, Netherlands. $94 \mathrm{pp}$.

Nagai, T.; Nagashima, T.; Myoda, T.; Inoue, R.

2004. Preparation and functional properties of extracts from bee bread. Food / Nahrung 48(3): 226-229.

Neveu, V.; Perez-Jiménez, J.; Vos, F.; Crespy, V., du Chaffaut, L.; Mennen, L.; Knox, C.; Eisner, R.; Cruz, J.; Wishart, D.; Scalbert, A.

2010. Phenol-explorer: an online comprehensive database on polyphenol contents in foods. Database 2010: bap024, doi: 10.1093/database/bap024.

Rubilar, M.; Pinelo, M.; Ihl, M.; Scheuerman, E.; Siniero, J.; Jos, M.

2006. Murta leaves (Ugni molinae Turcz.) as a source of antioxidant polyphenols. Journal of Agricultural and Food Chemistry 54: 59-64.

Singleton, V.; Rossi, J.

1965. Colorimetry of total phenolics with phosphomolybdicphosphotungstic acid reagents. American Journal of Enology and Viticulture 16: 144-158.

SPSS Inc.

2006. SPSS Base 15.0 user's guide. Chicago, IL, USA. 591 pp.

Stanciu, O.; Marghitas, L.; Dezmirean, D.

2008. A comparison of methods used to define the antioxidant capacity of bee pollen and beebread from Romania. En: 
Proceedings of the 43rd Croatian and 3rd International Symposium on Agriculture. Opatija, Criatia. pp. 751-754. Stanciu, O.; Marghitas, L.; Dezmirean, D.

2007. Examination of antioxidant capacity of beebread extracts by different complementary assays. Bulletin of University of Agricultural Sciences and Veterinary Medicine Cluj-Napoca. Animal Science and Biotechnologies 63(1-2): 204-207.

Tresserra-Rimbau, A.; Medina-Remón, A.; Pérez-Jiménez, J.; Martínez-González, M.A.; Covas, M.I.; Corella, D.; Salas-Salvadó, J.; Gómez-Gracia, E.; Lapetra, J.; Arós, F.; Fiol, M.; Ros, E.;
Serra-Majem, L.; Pintó, X.; Muñoz, M.A.; Saez, G.T.; RuizGutiérrez, V.; Warnberg, J.; Estruch, R.; Lamuela-Raventós, R.M. 2013. Dietary intake and major food sources of polyphenols in a Spanish population at high cardiovascular risk: The PREDIMED study. Nutrition, Metabolism \& Cardiovascular Diseases 23 (10):953-959.

Yao, L.; Jiang, Y.; Singanusong, R.; D’Arcy, B.; Datta, N.; Caffin, N.; Raymont, K.

2004. Flavonoid in Australian Malaleuca, Guioa, Lophostemon, Banksia and Helianthus honeys and their potential for floral authentication. Food Research International 37(2): 166-174. 
